\title{
U-shaped Association Between Apolipoprotein B and All-Cause Mortality: Results from the National Health and Nutrition Examination Surveys
}

mengqi yan

Guangdong Cardiovascular Institute

Xiaocong Liu

Guangdong Cardiovascular Institute

Yuqing Huang

Guangdong Cardiovascular Institute

Yuling Yu

Guangdong Cardiovascular Institute

Dan Zhou

Guangdong Cardiovascular Institute

Yingqing Feng ( $\square$ fyq1819@163.com )

Guangdong Cardiovascular Institute https://orcid.org/0000-0001-7662-327X

Research

Keywords: Apolipoprotein B, all-cause mortality, cardiovascular mortality, NHANES

Posted Date: November 9th, 2021

DOI: https://doi.org/10.21203/rs.3.rs-1039792/v1

License: (9) (i) This work is licensed under a Creative Commons Attribution 4.0 International License.

Read Full License 


\section{Abstract}

\section{Background}

Apolipoprotein $\mathrm{B}(\mathrm{apoB})$, a significant component directly reflecting the number of atherogenic lipoprotein particles, gradually becomes a more conducive indicator to control blood lipids. However, epidemiological evidence on its relationship with mortality is limited, especially with all-cause mortality.

\section{Methods}

Participants from the National Health and Nutrition Examination Survey during 2007-2014 were grouped according to the apoB quartiles (15-76, 77-92, 93-110, 111-345mg/dL). We performed Cox proportional hazards models and Kaplan-Meier survival curves to evaluate the relationships of apoB with all-cause and cardiovascular mortality. Restricted cubic spline and piecewise linear regression were performed to detect their non-linear relationships.

\section{Results}

In general, we enrolled 10375 participants among United States adults (mean age $46.3 \pm 16.9,47.88 \%$ men). On average, participants were followed up for 69.2 months, among whom 533 (5.14\%) and 91 $(0.88 \%)$ deaths were observed due to all -causes and cardiovascular diseases, respectively. After adjusting for confounders, apoB was independently associated with an elevated risk of cardiovascular death (hazard ratio [HR], 1.13; 95\% confidence interval [Cl], 1.03-1.24). However, in the third quartile of apoB, the risk of all-cause death decreased significantly ( $\mathrm{HR}, 0.71 ; 95 \% \mathrm{Cl}, 0.56-0.91)$. Moreover, the nonlinear relationship between apoB and all-cause death demonstrated an increased risk at both low and high level apoB concentrations, divided by the threshold point of $108 \mathrm{mg} / \mathrm{dl}$.

\section{Conclusion}

Elevated apoB was significantly associated with an increased risk for cardiovascular mortality, while its association with all-cause mortality was non-linear correlated, with an increased risk at both low and high apoB levels.

\section{Introduction}

Apolipoprotein $B(a p o B)$ is a significant component for atherogenic particles, including very-low-density lipoprotein (VLDL), chylomicrons, low-density lipoprotein ( $L D L)$, and intermediate-density lipoprotein (IDL), and each particle contains one molecule of Apo B (1). ApoB exists as two isoforms-- apoB-100 and apoB-48, originating from hepatocytes and the small intestine, respectively(2). Low-density lipoprotein cholesterol (LDL-C), a cornerstone measurement for guiding lipid-lowering therapy for nearly decades, does not always reflect LDL particle number precisely because of the wide variety of LDL-C concentration among individuals(3). Consequently, although LDL-C-targeted therapies are widely accepted, it is insufficient to measure the blood lipid level and risk factors of atherosclerotic cardiovascular disease 
(ASCVD) only by LDL-C(4). Meanwhile, more than $90 \%$ of apoB in plasma is apo B-100 associated with LDL, leading apoB a good alternate for LDL particle concentration, especially in patients with cholesterolenriched apoB particles (5). Therefore, the assessment of apoB directly reflects the number of atherogenic lipoprotein particles in the circulation. Moreover, focuses on this indicator is more conducive to controlling blood lipids $(6,7)$.

Previous studies have demonstrated that apoB and ASCVD are more closely related $(8,9)$. However, epidemiological evidence on its relationship with all-cause mortality and cardiovascular death is limited. Therefore, this study aimed to address the knowledge gap of apoB with cardiovascular and all-cause mortality among United States adults in the National Health and Nutrition Examination Surveys (NHANES)

\section{Materials And Methods}

Study Design and Participants

NHANES is a cross-sectional, nationally representative survey that gathers data on the nutrition and health status of the non-institutionalized US population of all ages, conducted by the Centers for Disease Control and Prevention (CDC). This study was based on the NHANES in 2007-2014 (http://wwwn.cdc.gov/nchs/nhanes). After excluding those with CVD or cancer at baseline, a total of 10375 eligible participants with complete apoB and follow-up data were included in this analysis (Figure 1). The Institutional Review Board approved the survey protocol of the CDC. All participants have provided written informed consent.

Measurement of apoB

ApoB data were available for only those who provided their blood samples in the morning session in a fasting state. ApoB was measured by immunonephelometric assays using a ProSpec Nephelometer (Siemens GMBH. Marburg, Germany D-35041) at the University of Minnesota Medical Center. The immunonephelometric method uses polyclonal antibodies specific to apoB, in which the intensity of the scattered light is directly proportional to the concentration of $a p o B$ in the specimen. Besides, the apoB concentration of the test specimen is determined by comparing its light scatter to that observed using known standards in a stored calibration curve. In this survey, the unit of apoB is $\mathrm{mg} / \mathrm{dl}$. During the 1980 s and 1990s, the International Federation for Clinical Chemistry (IFCC) reached a collaborative initiative with various diagnostic companies, determining the international standardization and harmonization of apoB methods named WHO/IFCC SP3-07(10-12). Examinations of apoB in this study meet the standards established by this meeting. More details are accessible at http://cdc.gov/nchs/nhanes under the lab methods section.

Covariates and Outcomes 
Using standardized questionnaires, participants provided information on age (years), sex (male, female), race (classify as white or other), smoking (classify as yes or no), an education level (less than high school, high school, or above) and self-reported medical history. Examinations of height, weight, and body mass index (BMI) were measured by anthropometric measurement. The estimated glomerular filtration rate (eGFR) was calculated by the Modification of Diet in Renal Disease (MDRD) formula(13). Hypertension were defined as systolic blood pressures (SBP) $\geq 140 \mathrm{mmHg}$ and/or diastolic blood pressure (DBP) $\geq 90 \mathrm{mmHg}$, reported the usage of hypotensive drugs, or history of hypertension(14). Diabetes Mellitus was defined as fasting blood glucose $\geq 126 \mathrm{mg} / \mathrm{dl}$, hemoglobin A1c $\geq 6.5 \%$, reported the usage of hypoglycemic drugs, or history of diabetes(15). According to a standardized procedure and protocol, other covariates included total cholesterol (TC), triglyceride (TG), high-density lipoprotein cholesterol (HDL-C), and low-density lipoprotein cholesterol (LDL-C). If TG was $\leq 400 \mathrm{mg} / \mathrm{dL}, \mathrm{LDL}-\mathrm{C}$ was computed by the Friedewald formula(16). Outcomes in this study were all-cause and cardiovascular mortality. And all mortality data were obtained from a publicly available dataset named National Death Index (NDI), which included participants' data and linked with the NHANES dataset. The definition of cardiovascular mortality was adapted from the International Classification of Diseases, 10th Clinical Modification (ICD-10) System codes (I00-I09, I11, I13, I20-I51, I60-I69)(17).

Statistical analysis

We grouped participants according to the apoB quartiles $(15-76,77-92,93-110,111-345 \mathrm{mg} / \mathrm{dL})$ in baseline characteristics. Accordingly, continuous variables are presented as mean \pm standard deviation and categorical variables as number with percentage. Depending on the nature of the data, we used Chisquare test, Kruskal-Wallis H-test, or ANOVA to detect subgroup differences. Three sets of cox regression models were performed to investigate the association between apoB and mortality. Model 1 only included apoB as a continuous variable or quartiles. Model 2 was adjusted for age, sex, and race. In addition to the variables in Model 2, smoking status, education level, BMI, SBP, eGFR, TG, HDL-C, energy intake, diabetes, hypertension, antihypertensive drugs, hypoglycemic drugs, and lipid-lowering medication were additionally adjusted in Model 3 . Besides, a trend across apoB quartile groups was tested. Kaplan-Meier survival analyses were performed to present all-cause and cardiovascular mortality by apoB quartiles, and the log-rank test compared survival probabilities. The non-linear relationship between apoB and mortality was detected using restricted cubic spline models. Two-piecewise linear regression models were applied to determine the threshold point for the non-linear relationship. We further employ a logarithmic likelihood ratio test to compare the differences between one-line linear regression models and twopiecewise linear regression models. All statistical analyses were performed with $R$ version 3.6.3 ( $R$ Foundation for Statistical Computing, Vienna, Austria). Statistical significance was accepted at the level of $\mathrm{P}<0.05$.

\section{Results}

Baseline Characteristics 
Sociodemographic, laboratory, and clinical characteristics at baseline of 10375 participants are shown in Table 1. Among those participants (mean age $46.3 \pm 16.9,47.88 \%$ men, the average apoB was 93.76 (SD, $25.82) \mathrm{mg} / \mathrm{dL}$. With an average follow-up time of 69.2 months, $533(5.14 \%)$ death occurred totally, of which $91(0.88 \%)$ died of cardiovascular diseases during the follow-up period. Higher apoB quartiles were related with males, lower education level, higher BMI and blood lipid level, and comorbidities with hypertension $(P<0.001)$. Compared to the lower quartiles, individuals in the highest apoB quartile were more likely to have comorbid diabetes (19.23\%).

The Relationship of ApoB With All-Cause and Cardiovascular Mortality.

Stratified by apoB quartiles (15-76, 77-92, 93-110, 111-345 mg/dL), survival probabilities displayed in Kaplan-Meier curves were demonstrated in Figure 2. The accumulative incidence of cardiovascular mortality increased with apoB (log-rank test, $P=0.04$ ) while there were no statistical differences among apoB quartile groups for all-cause mortality (log-rank test, $P=0.72$ ). As shown in Table 2 , when using the value of apoB as a continuous variable, apoB was positively correlated (HR, 1.13; 95\% Cl, 1.03-1.24) with cardiovascular death events and statistically irrelevant $(\mathrm{HR}, 0.97 ; 95 \% \mathrm{Cl}, 0.93-1.01)$ with all-cause mortality in fully adjusted model. Similarly, when using apoB quartiles as categorical variables, the trend between elevating apoB and all-cause mortality was not significant compared to the reference group (the lowest apoB quartile). However, the third quartile indicated a notable decline in all-cause death after adjusting for confounders ( $\mathrm{HR}, 0.71 ; 95 \% \mathrm{Cl}, 0.56,0.91)$. Meanwhile, participants in higher apoB quartiles had increased risks of cardiovascular death ( $P$ for trend $<0.0053$ ) in Model I, especially in the highest apoB group (HR, 2.45; 95\% Cl, 1.29-4.65). The positive correlation with cardiovascular mortality persisted in Model III ( $P$ for trend $<0.0412)$.

The Analyses of Non-linear Relationship

Restricted cubic spline models were applied to analyze the non-linear relationship between apoB and mortality in Figure 3. Results implied that there was a U-shaped association between apoB and all-cause mortality (Non-linear $\mathrm{P}=0.005$ ), but not between apoB and cardiovascular death (Non-linear $\mathrm{P}=0.88$ ). According to two piecewise linear regression models (Table 3), the threshold value was $108 \mathrm{mg} / \mathrm{dL}$ for allcause mortality. Below the threshold point, apoB was negatively associated with cardiovascular mortality (HR, $0.90 ; 95 \% \mathrm{Cl}, 0.85-0.95)$, while above the threshold point, the increment of apoB was positively related with cardiovascular mortality $(\mathrm{HR}, 1.09 ; 95 \% \mathrm{Cl}, 1.01-1.19)$.

\section{Discussion}

Our study found that participants in the fourth quartile had more frequent comorbidities with diabetes mellitus at baseline characteristics. When treating $a p o B$ as a continuous variable, the HR for cardiovascular mortality per $1 \mathrm{mg} / \mathrm{dL}$ increase in apoB was 1.13 . Furthermore, a U-shaped association was shown in apoB and all-case motality, with the higher risks of all-cause mortality on both sides of apoB levels, in which the threshold value was $108 \mathrm{mg} / \mathrm{dL}$. 
The European Society of Cardiology (ESC) and European Atherosclerosis Society (EAS) stated that apoB should be a secondary target of preventive therapy in patients with hypertriglyceridemia, obesity, or diabetes according to cardiovascular mortality risk categories(18). Among the above patients, accumulating small lipid-depleted LDL subfractions containing less cholesterol is produced, leading to high apoB concentration without necessarily high LDL-C(3). Furthermore, targeting only LDL-C could result in missing opportunities for CV risk reduction in type 2 diabetes mellitus (T2DM) patients(19). These researches are consistent with our finding that the fourth quartile had more frequent comorbidities with diabetes, indicating that apoB provides a more consistent goal for statin treatment than the LDL$\mathrm{C}(20)$.

We found that apoB was positively correlated with cardiovascular mortality even after adjusting for the confounders. Zhu et al.(21) also found that ApoB was associated with occurrence rates of adverse cardiovascular events $(\mathrm{HR}, 1.349 ; 95 \% \mathrm{Cl}, 1.073-1.695)$. The findings of our study were not similar to previous studies on the relationship between LDL-C and cardiovascular deaths. In meta-analyses, Navarese et al.(22) claimed that the most significant reduction in risk of all-cause and cardiovascular mortality from LDL-C-lowering therapy might occur for patients with higher baseline LDL-C levels not for lower LDL-C levels (less than $100 \mathrm{mg} / \mathrm{dL}$ ). Other researches also indicated that LDL-C did not perform well at low concentrations, regardless of the relationship with all-cause or cardiovascular mortality (23, 24). Additionally, Mendelian randomization studies had reported that the reduction in cardiovascular risk was associated with lowering the number of atherogenic apoB particles rather than the mass of cholesterol as measured by LDL-C(25), which was consistent with our results. Generally speaking, although LDL-C still acts as the primary criterion for lowering blood lipids in clinical practice, apoB, a more sensitive indicator, should be used more frequently for guiding lipid-lowering treatment, especially for cardiovascular events.

A critical finding of our study was that the association between levels of apoB and all-cause mortality was U-shaped, with low and high levels associated with an increased risk of all-cause mortality. This result was different from the trend of cardiovascular mortality. Besides, when apoB was used as a categorical variable, there was a statistically significant decrease in all-cause mortality in the Q3 group after adjusting confounders, which was consistent with the non-linear relationship. This finding was contrary to the general assumption that higher mortality always along with high lipoprotein levels. However, numerous studies performed different associations between lipoprotein levels and all-cause mortality. From the Copenhagen General Population Study, Camilla et al.(9) found a J-shaped association between apoB and all-cause mortality among patients receiving statin therapy. Similarly, in the same study population, researchers showed a U-shaped relation between LDL-C and HDL-C and all-cause mortality levels, even after being adjusted for baseline comorbidities $(26,27)$. However, most previous studies on the association between LDL-C and mortality risk showed no associations or inverse associations(28-30). Although we had adjusted some confounders, whether the association between moderately increased apoB levels and decreased mortality is causal is still an unresolved question to the findings. Researchers found that, compared to healthy individuals, chronic obstructive pulmonary disease (COPD) patients had higher HDL-C and lower TG, TC, and LDL-C, while those lipid parameters varied 
reversely in COPD patients with cardiovascular diseases(31). Other disease conditions, including debilitation and wasting in liver diseases, would result in less secretion of lipoproteins(32). Furthermore, these conditions have been hypothesized to cause a decrease in levels of apoB. Besides, discordance analysis demonstrated that apoB was a more accurate marker of all-cause mortality risk in statin-treated patients than LDL-C or non-HDL cholesterol(9). Generally speaking, apoB, a more sensitive indicator, should be discussed and applied more frequently for guiding lipid-lowering treatment in clinical practice.

Another critical finding of this study was that we disclosed the threshold value (108 $\mathrm{mg} / \mathrm{dL})$ for all-cause mortality according to two piecewise linear regression models. Nonetheless, the threshold value was 73 $\mathrm{mg} / \mathrm{dl}$ in the Camilla et al.(9) study, in which the median value for apoB was consistent with our study (92 $\mathrm{mg} / \mathrm{dl})$. The reasons for inconsistent thresholds may be related to differences in adjusted variables and study populations. Currently, the standard of $a p o B>130 \mathrm{mg} / \mathrm{dl}$ multi-society endorsed by the American College of Cardiology (ACC) and American Heart Association (AHA) for patients with an average estimated risk score seems inappropriately high compared with the threshold $(108 \mathrm{mg} / \mathrm{dl})$ of our study(33). In contrast, ESC/EAS guideline defines the secondary target for apoB as $<100 \mathrm{mg} / \mathrm{dl}$ in patients with mild-to-moderate hypertriglyceridemia $(175-880 \mathrm{mg} / \mathrm{dL})$, including those with obesity or metabolic syndrome, diabetes, or chronic kidney disease seem more reasonable(18).

Despite the critical findings we mentioned above, several limitations should be taken into consideration. There might be restrictions regarding generalization across other regions as we only included the United States citizens. Another limitation is that our study did not imply causality between apoB-lowering treatment and decreased mortality due to the cross-sectional nature. Further randomized clinical trials (RCTs) should be conducted to confirm this perspective.

\section{Conclusion}

There were differences in the association between apoB with cardiovascular and all-cause mortality among the US population. ApoB was significantly associated with an increased risk for cardiovascular mortality, while there existed a non-linear relationship of apoB with all-cause mortality with an increased risk of all-cause mortality at both low and high apoB levels. Whether the reduction of apoB may reduce cardiovascular and all-cause mortality risk, in the long run, needs to be further evaluated by intervention studies.

\section{Declarations}

\section{Ethics approval and consent to participate}

The survey protocol was approved by the Institutional Review Board of the Centers for Disease Control and Prevention (Protocol 98-12, 2005-06 and 2011-17). All patients received informed consent before the start of the study.

\section{Consent for publication}


All authors approved and agreed to publish the final version of the manuscript.

\section{Availability of data and materials}

Data are from the NHANES Study. Data are available in a public, open access repository.

\section{Competing interests}

The authors declare that they have no competing interests.

\section{Funding}

This work was supported by the Science and Technology Plan Program of Guangzhou (No. 201803040012), the Key Area R\&D Program of Guangdong Province (No. 2019B020227005) and the National Key Research and Development Program of China (No.2017YFC1307603).

\section{Authors' contributions}

Meng-qiYan and Xiao-cong Liu contributed to data analysis and manuscript drafting. Yu-qing Huang and Xiaocong-Liu contributed to data downloading. All authors contributed to manuscript revising and have approved the final article.

\section{Acknowledgements}

Not applicable.

\section{Authors' information}

1 Department of Cardiology, Guangdong Cardiovascular Institute, Guangdong Provincial People's Hospital, Guangdong Academy of Medical Sciences, Guangzhou, Guangdong, China

\section{References}

1. Kane JP. Apolipoprotein B: structural and metabolic heterogeneity. Annual review of physiology. 1983;45:637-50. Epub 1983/01/01. doi: 10.1146/annurev.ph.45.030183.003225. PubMed PMID: 6342527.

2. Elovson J, Chatterton JE, Bell GT, Schumaker VN, Reuben MA, Puppione DL, et al. Plasma very low density lipoproteins contain a single molecule of apolipoprotein B. Journal of lipid research. 1988;29(11):1461-73. Epub 1988/11/01. PubMed PMID: 3241122.

3. Sniderman AD, Thanassoulis G, Glavinovic T, Navar AM, Pencina M, Catapano A, et al. Apolipoprotein B Particles and Cardiovascular Disease: A Narrative Review. JAMA cardiology. 2019;4(12):1287-95. Epub 2019/10/24. doi: 10.1001/jamacardio.2019.3780. PubMed PMID: 31642874; PubMed Central PMCID: PMCPMC7369156. 
4. Sinning D, Landmesser U. Is There a Need to Revise Goals in the Management of Dyslipidemias? Current cardiology reports. 2019;21(6):51. Epub 2019/05/02. doi: 10.1007/s11886-019-1128-6. PubMed PMID: 31041621.

5. Contois JH, McConnell JP, Sethi AA, Csako G, Devaraj S, Hoefner DM, et al. Apolipoprotein B and cardiovascular disease risk: position statement from the AACC Lipoproteins and Vascular Diseases Division Working Group on Best Practices. Clinical chemistry. 2009;55(3):407-19. Epub 2009/01/27. doi: 10.1373/clinchem.2008.118356. PubMed PMID: 19168552.

6. Packard CJ. Determinants of Achieved LDL Cholesterol and "Non-HDL" Cholesterol in the Management of Dyslipidemias. Current cardiology reports. 2018;20(8):60. Epub 2018/06/16. doi: 10.1007/s11886-018-1003-x. PubMed PMID: 29904807.

7. Sandesara PB, Virani SS, Fazio S, Shapiro MD. The Forgotten Lipids: Triglycerides, Remnant Cholesterol, and Atherosclerotic Cardiovascular Disease Risk. Endocrine reviews. 2019;40(2):537-57. Epub 2018/10/13. doi: 10.1210/er.2018-00184. PubMed PMID: 30312399; PubMed Central PMCID: PMCPMC6416708.

8. Sniderman AD, Lamarche B, Contois JH, de Graaf J. Discordance analysis and the Gordian Knot of LDL and non-HDL cholesterol versus apoB. Current opinion in lipidology. 2014;25(6):461-7. Epub 2014/10/24. doi: 10.1097/mol.0000000000000127. PubMed PMID: 25340478.

9. Johannesen CDL, Mortensen MB, Langsted A, Nordestgaard BG. Apolipoprotein B and Non-HDL Cholesterol Better Reflect Residual Risk Than LDL Cholesterol in Statin-Treated Patients. Journal of the American College of Cardiology. 2021;77(11):1439-50. Epub 2021/03/20. doi:

10.1016/j.jacc.2021.01.027. PubMed PMID: 33736827.

10. Albers JJ, Marcovina SM, Kennedy H. International Federation of Clinical Chemistry standardization project for measurements of apolipoproteins A-I and B. II. Evaluation and selection of candidate reference materials. Clinical chemistry. 1992;38(5):658-62. Epub 1992/05/01. PubMed PMID: 1582016.

11. Marcovina SM, Albers JJ, Kennedy H, Mei JV, Henderson LO, Hannon WH. International Federation of Clinical Chemistry standardization project for measurements of apolipoproteins A-I and B. IV. Comparability of apolipoprotein B values by use of International Reference Material. Clinical chemistry. 1994;40(4):586-92. Epub 1994/04/01. PubMed PMID: 8149615.

12. Albers JJ, Marcovina SM. Standardization of apolipoprotein B and A-I measurements. Clinical chemistry. 1989;35(7):1357-61. Epub 1989/07/01. PubMed PMID: 2503261.

13. Inker LA, Schmid $\mathrm{CH}$, Tighiouart $\mathrm{H}$, Eckfeldt JH, Feldman $\mathrm{HI}$, Greene $\mathrm{T}$, et al. Estimating glomerular filtration rate from serum creatinine and cystatin $C$. The New England journal of medicine. 2012;367(1):20-9. Epub 2012/07/06. doi: 10.1056/NEJMoa1114248. PubMed PMID: 22762315; PubMed Central PMCID: PMCPMC4398023. 
14. Liu Y. National Health and Nutrition Examination Survey 1999-2000: effect of observer training and protocol standardization on reducing blood pressure measurement error. Journal of clinical epidemiology. 2004;57(6):651; author reply 2. Epub 2004/07/13. doi: 10.1016/j.jclinepi.2004.02.001. PubMed PMID: 15246137.

15. Petersmann A, Müller-Wieland D, Müller UA, Landgraf R, Nauck M, Freckmann G, et al. Definition, Classification and Diagnosis of Diabetes Mellitus. Experimental and clinical endocrinology \& diabetes : official journal, German Society of Endocrinology [and] German Diabetes Association. 2019;127(S 01):S1s7. Epub 2019/12/21. doi: 10.1055/a-1018-9078. PubMed PMID: 31860923.

16. Scharnagl H, Nauck M, Wieland H, März W. The Friedewald formula underestimates LDL cholesterol at low concentrations. Clinical chemistry and laboratory medicine. 2001;39(5):426-31. Epub 2001/07/04. doi: 10.1515/cclm.2001.068. PubMed PMID: 11434393.

17. Disantostefano JJJfNP. International Classification of Diseases 10th Revision (ICD-10). 2009;5(1):56-7.

18. Mach F, Baigent C, Catapano AL, Koskinas KC, Casula M, Badimon L, et al. 2019 ESC/EAS Guidelines for the management of dyslipidaemias: lipid modification to reduce cardiovascular risk. European heart journal. 2020;41(1):111-88. Epub 2019/09/11. doi: 10.1093/eurheartj/ehz455. PubMed PMID: 31504418.

19. Fonseca L, Paredes S, Ramos H, Oliveira JC, Palma I. Apolipoprotein B and non-high-density lipoprotein cholesterol reveal a high atherogenicity in individuals with type 2 diabetes and controlled lowdensity lipoprotein-cholesterol. Lipids in health and disease. 2020;19(1):127. Epub 2020/06/09. doi: 10.1186/s12944-020-01292-w. PubMed PMID: 32505210; PubMed Central PMCID: PMCPMC7275418.

20. Charlton-Menys V, Betteridge DJ, Colhoun H, Fuller J, France M, Hitman GA, et al. Targets of statin therapy: LDL cholesterol, non-HDL cholesterol, and apolipoprotein B in type 2 diabetes in the Collaborative Atorvastatin Diabetes Study (CARDS). Clinical chemistry. 2009;55(3):473-80. Epub 2009/01/17. doi: 10.1373/clinchem.2008.111401. PubMed PMID: 19147732.

21. Zhu YM, Verma S, Fung M, McQueen MJ, Anderson TJ, Lonn EM. Association of Apolipoproteins B and A-1 With Markers of Vascular Health or Cardiovascular Events. The Canadian journal of cardiology. 2017;33(10):1305-11. Epub 2017/09/25. doi: 10.1016/j.cjca.2017.08.004. PubMed PMID: 28941609.

22. Navarese EP, Robinson JG, Kowalewski M, Kolodziejczak M, Andreotti F, Bliden K, et al. Association Between Baseline LDL-C Level and Total and Cardiovascular Mortality After LDL-C Lowering: A Systematic Review and Meta-analysis. 2018;319(15):1566.

23. Tsujimoto T, Kajio H, Sugiyama T. Statin Therapy in Patients With Low Serum Levels of Low-Density Lipoprotein Cholesterol. The American journal of cardiology. 2017;120(11):1947-54. Epub 2017/09/28. doi: 10.1016/j.amjcard.2017.08.011. PubMed PMID: 28951019. 
24. Leibowitz M, Karpati T, Cohen-Stavi CJ, Feldman BS, Hoshen M, Bitterman H, et al. Association Between Achieved Low-Density Lipoprotein Levels and Major Adverse Cardiac Events in Patients With Stable Ischemic Heart Disease Taking Statin Treatment. JAMA internal medicine. 2016;176(8):1105-13. Epub 2016/06/21. doi: 10.1001/jamainternmed.2016.2751. PubMed PMID: 27322095.

25. Ference BA, Kastelein J, Ginsberg HN, Chapman MJ, Nicholls SJ, Ray KK, et al. Association of Genetic Variants Related to CETP Inhibitors and Statins With Lipoprotein Levels and Cardiovascular Risk. 2017;318(10):947.

26. Johannesen CDL, Langsted A, Mortensen MB, Nordestgaard BG. Association between low density lipoprotein and all cause and cause specific mortality in Denmark: prospective cohort study. BMJ (Clinical research ed). 2020;371:m4266. Epub 2020/12/10. doi: 10.1136/bmj.m4266. PubMed PMID: 33293274; PubMed Central PMCID: PMCPMC7722479

27. Madsen CM, Varbo A, Nordestgaard BG. Extreme high high-density lipoprotein cholesterol is paradoxically associated with high mortality in men and women: two prospective cohort studies. European heart journal. 2017;38(32):2478-86. Epub 2017/04/19. doi: 10.1093/eurheartj/ehx163. PubMed PMID: 28419274.

28. Bathum L, Depont Christensen R, Engers Pedersen L, Lyngsie Pedersen P, Larsen J, Nexøe J. Association of lipoprotein levels with mortality in subjects aged $50+$ without previous diabetes or cardiovascular disease: a population-based register study. Scandinavian journal of primary health care. 2013;31(3):172-80. Epub 2013/08/15. doi: 10.3109/02813432.2013.824157. PubMed PMID: 23941088; PubMed Central PMCID: PMCPMC3750440.

29. Psaty BM, Anderson M, Kronmal RA, Tracy RP, Orchard T, Fried LP, et al. The association between lipid levels and the risks of incident myocardial infarction, stroke, and total mortality: The Cardiovascular Health Study. Journal of the American Geriatrics Society. 2004;52(10):1639-47. Epub 2004/09/29. doi: 10.1111/j.1532-5415.2004.52455.x. PubMed PMID: 15450039.

30. Jeong SM, Choi S, Kim K, Kim SM, Lee G, Son JS, et al. Association of change in total cholesterol level with mortality: A population-based study. PloS one. 2018;13(4):e0196030. Epub 2018/04/20. doi: 10.1371/journal.pone.0196030. PubMed PMID: 29672595; PubMed Central PMCID: PMCPMC5908176.

31. Markelić I, Hlapčić I, Rogić D, Rako I, Samaržija M, Popović-Grle S, et al. Lipid profile and atherogenic indices in patients with stable chronic obstructive pulmonary disease. Nutrition, metabolism, and cardiovascular diseases : NMCD. 2021;31(1):153-61. Epub 2020/09/29. doi:

10.1016/j.numecd.2020.07.039. PubMed PMID: 32981798.

32. Jacobs D, Blackburn H, Higgins M, Reed D, Iso H, McMillan G, et al. Report of the Conference on Low Blood Cholesterol: Mortality Associations. Circulation. 1992;86(3):1046-60. Epub 1992/09/01. doi: 10.1161/01.cir.86.3.1046. PubMed PMID: 1355411. 
33. Grundy SM, Stone NJ, Bailey AL, Beam C, Birtcher KK, Blumenthal RS, et al. 2018 AHA/ACC/AACVPR/AAPA/ABC/ACPM/ADA/AGS/APhA/ASPC/NLA/PCNA Guideline on the Management of Blood Cholesterol: A Report of the American College of Cardiology/American Heart Association Task Force on Clinical Practice Guidelines. Circulation. 2019;139(25):e1082-e143. Epub 2018/12/28. doi:

10.1161/cir.0000000000000625. PubMed PMID: 30586774; PubMed Central PMCID: PMCPMC7403606.

\section{Tables}

Due to technical limitations, table 1, 2 and 3 is only available as a download in the Supplemental Files section.

\section{Figures}

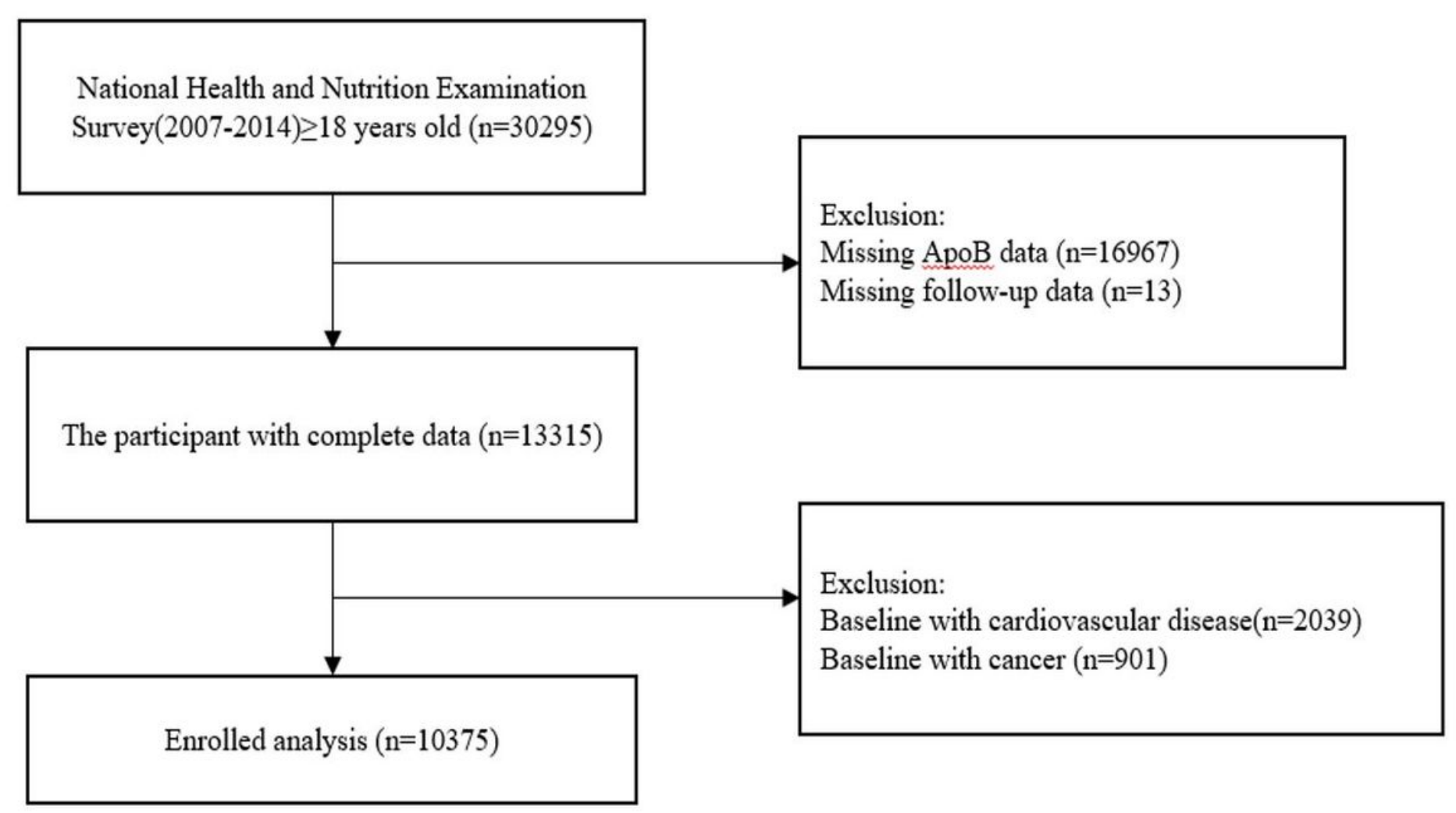

Figure 1

Study cohort. 
A

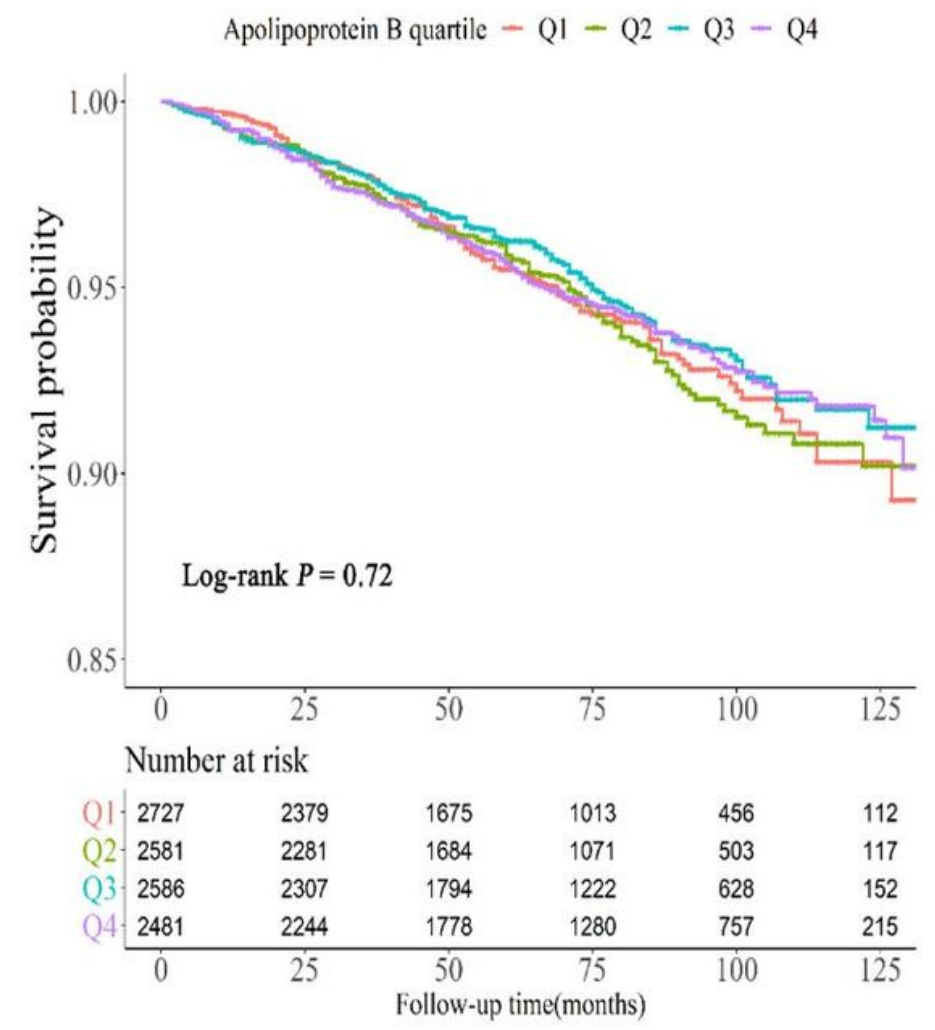

B

Apolipoprotein B quartile - Q1 - Q2 - Q3 - Q4

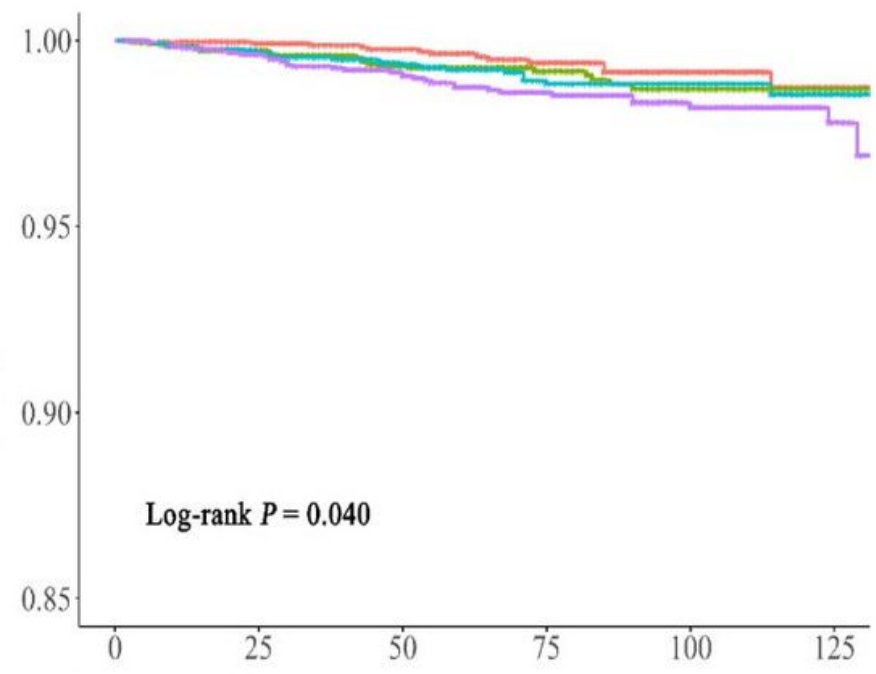

Number at risk

\begin{tabular}{|cccccc}
2727 & 2379 & 1675 & 1013 & 456 & 112 \\
2581 & 2281 & 1684 & 1071 & 503 & 117 \\
2586 & 2307 & 1794 & 1222 & 628 & 152 \\
2481 & 2244 & 1778 & 1280 & 757 & 215 \\
\hline 0 & 25 & 50 & 75 & 100 & 125 \\
& & Follow-up time(months) &
\end{tabular}

\section{Figure 2}

Kaplan-Meier survival curves for all-cause (A) and cardiovascular (B) mortality by Apolipoprotein B quartiles.

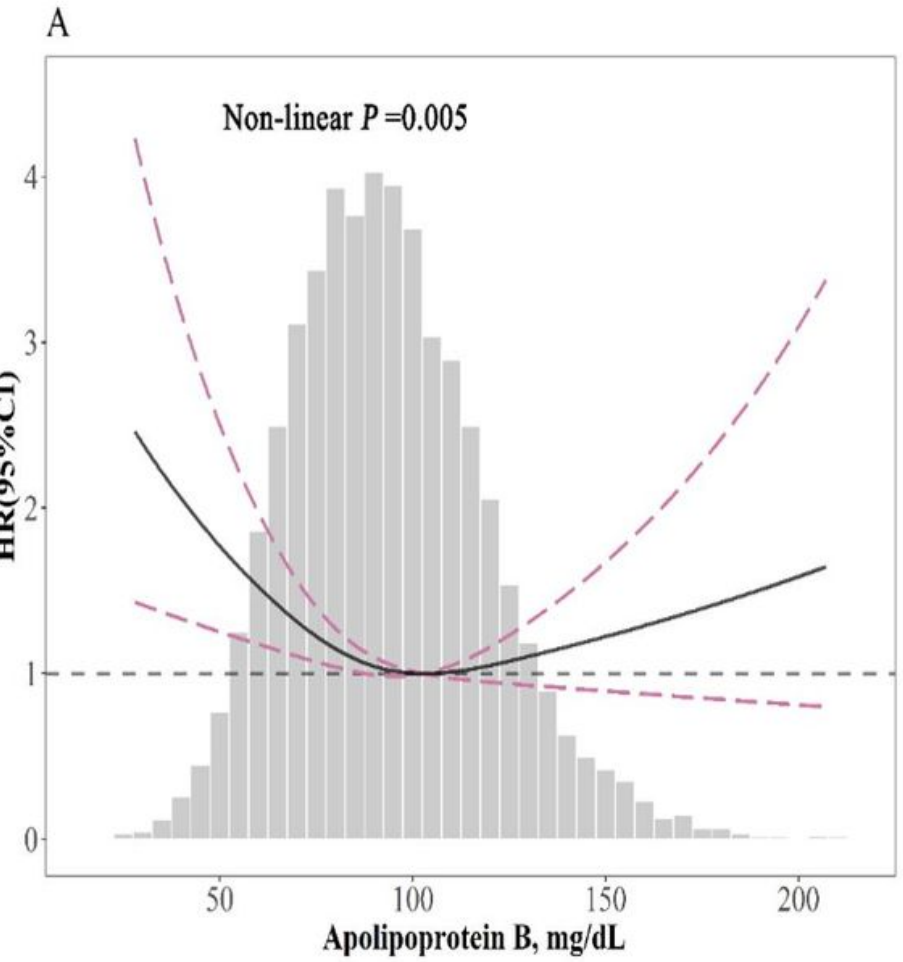

B

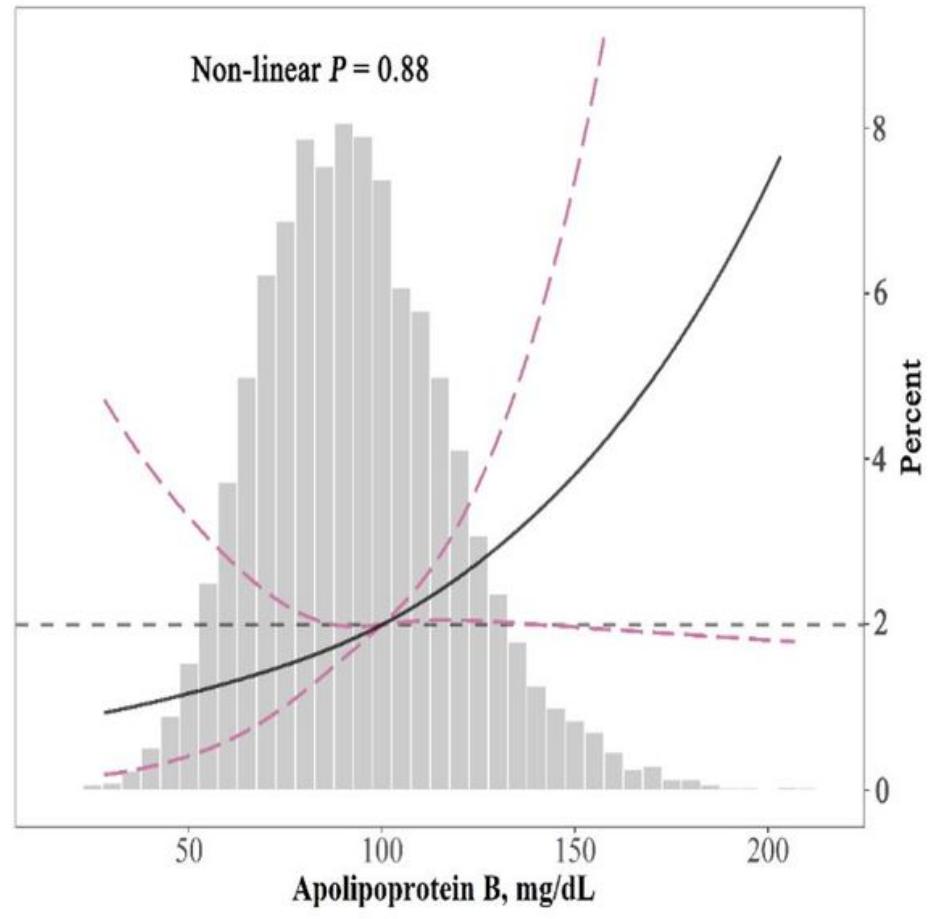




\section{Figure 3}

Spline analyses of all-cause (A) and cardiovascular (B) mortality by Apolipoprotein B in the overall cohort, and the probability distribution histogram is represented in the background. (Spline analyses were adjusted for age, gender, race, smoking, education level, body mass index, systolic blood pressure, estimated glomerular filtration rate, triglyceride, high-density lipoprotein cholesterol, energy intake, diabetes and hypertension, antihypertensive drugs, hypoglycemic agents, and lipid-lowering drugs).

\section{Supplementary Files}

This is a list of supplementary files associated with this preprint. Click to download.

- table1.pptx

- Table2.pptx

- Table3.pptx 